\title{
Characteristic Alterations in Adrenal Catecholamine Contents in SHR, SHRSP, and WKY during Development of Hypertension and Stroke
}

\author{
Shunichi Maemura, M.S., Masami Niwa, M.D., \\ and Masayori Ozaki, M.D.
}

\section{SUMmary}

The role of adrenal catecholamines (CAs) was investigated with regard to the etiology of hypertension and cerebral stroke in the spontaneously hypertensive rats-stroke prone (SHRSP). The adrenal CAs in SHRSP were measured by high performance liquid chromatography with an electrochemical detector or by gas-liquid chromatography with an electron capture detector and the findings were compared with those in the spontaneously hypertensive rats (SHR) and Wistar-Kyoto strain (WKY). It has been proposed that the facilitation of peripheral sympathetic norepinephrine (NE) neurons in the young animals may act as a trigger in the development of hypertension in the SHR. This was verified by estimating the adrenal NE contents in both SHRSP and SHR at 4 weeks of age. A deficiency in adrenal dopamine (DA) in 4-week-old SHRSP was also observed. This deficiency may contribute to the facilitation of the adrenal NE cell. SHRSP was clearly distinguished from SHR by comparing the adrenal catecholamine contents of each strain. The contents of all three CAs in SHRSP were similar to those in WKY during the development of hypertension, while the contents of epinephrine and DA in the SHR were much higher than those in the WKY. Only in SHRSP did the contents of all three CAs increase rapidly after the development of hypertension. These rapid increases may be related to stroke.

\section{Additional Indexing Words:}

Adrenal dopamine Adrenal norepinephrine Adrenal epinephrine HPLC-EC

From the Second Department of Pharmacology, Nagasaki University School of Medicine, Nagasaki, Japan.

This study was supported by the Grant No. 56770129 (1981) from the Ministry of Education, Science, and Culture, Japan.

Address for reprint: Masayori Ozaki, M.D., Second Department of Pharmacology, Nagasaki University School of Medicine, 12-4 Sakamoto-machi, Nagasaki 852, Japan.

Received for publication October 5, 1981. 
$7 \mathrm{HE}$ role of the central and the peripheral catecholaminergic structure in experimental hypertension, particulary in the spontaneously hypertensive rats (SHR), has been investigated extensively. In SHR, the facilitation of the peripheral sympathetic nervous system, as related to central abnormalities, is considered to be closely related to the development of hypertension. Here, the adrenal medulla is one of the most important peripheral sympathetic organs and is a major source of the circulating catecholamines (CAs) in rats. Nagatsu et al" reported that both young and adult SHR had higher activities of catecholamine (CA) synthesizing enzymes in the adrenal grands than did age matched normotensive Wistar rats (NWR). Morisawa ${ }^{2}$ ) reported that the areas of norepinephrine (NE)-storing cell islets in the adrenal medulla of young and adult SHR were twice as large as those of WistarKyoto strain (WKY). Ozaki et al ${ }^{3)}$ and others ${ }^{4}$ ) reported the NE content in SHR was twice as high as in WKY. However, there are conflicting reports with regard to epinephrine (E) contents of SHR and WKY. ${ }^{3), 4}$ There are few reports on the dopamine (DA) levels in the adrenal glands of SHR, as separation from other CAs in these tissues is most difficult. In one of few observations on the adrenal medulla in the SHR-stroke prone (SHRSP), Nagatsu et al $^{51}$ reported that the tyrosine hydroxylase activity in SHRSP at 16 weeks of age was higher than in SHR. In SHR, the inhibitors of CA synthesizing enzymes have a more pronounced hypotensive effect than is seen in NWR ${ }^{1,6)}$ when these activities are higher in the adrenal glands in SHR than in NWR. On the other hand, performance of bilateral adrenal demedullation before the onset of hypertension in SHR does not delay the onset. ${ }^{\text {) }}$ Accordingly, the role of adrenal medullary CAs in the development and the maintenance of hypertension in SHR and SHRSP remains unclear. With recent developments in sensitive assay methods for CAs, such as a gas-liquid chromatograph with an electron capture detector (GLC-ECD), ${ }^{8)}$ a radio enzymatic assay ${ }^{9 /}$ and high performance liquid chromatograph with an electrochemical detector (HPLC-EC), ${ }^{10)} \mathrm{E}, \mathrm{NE}$, and DA can be simultaneously determined in various peripheral tissues. In the present studies, we measured the CA contents, including DA, of the adrenal glands before, during and after the development of hypertension or stroke in SHRSP, using mainly HPLC-EC. These levels were then compared with those of SHR and WKY. The roles of the adrenal CAs in the development of hypertension and stroke were also examined. 


\section{Materials AND Methods}

\section{Animals}

Groups of 5 male SHRSP, SHR, and WKY rats, aged 4, 7, 10, 15, 20, and 30 weeks, were bred in our own laboratory. Room temperature was amintained at $23 \pm 1{ }^{\circ} \mathrm{C}$ and humidity at $55 \pm 10 \%$, and all rats were fed the usual commercial diet (Funahashi Farm Co.).

\section{Measurement of blood pressure}

The systolic blood pressure (SBP) was measured in the tail of conscious rats, 2 days before decapitation, with an electrosphygmomanometer (Narco Bio-Systems, Houston, Tex.). The rats were warmed for 10-15 min (38 $2^{\circ} \mathrm{C}$ ) before the measurement.

\section{Assay of adrenal catecholamines}

The rats were decapitated between 8 and 9 a.m. under minimum stress. After decapitation, left and right adrenal glands were quickly removed, cleaned, and weighed. The adrenal glands of the rats 4 weeks of age were homogenized in $5 \mathrm{ml}$ of ice cold $0.4 \mathrm{~N}$ perchloric acid containing $0.05 \%$ EDTA-2Na; those of other rats were homogenized in $8 \mathrm{ml}$ of the same medium. The homogenates were centrifuged at 20,000 $\mathrm{g}$ for $15 \mathrm{~min}$ in the cold and the deproteinized supernatants were stored at $-25^{\circ} \mathrm{C}$ until assayed.

The CAs were assayed by HPLC-EC in most of the adrenal samples, but GLG-ECD was used for the remaining. When the same supernatant sample was assayed by these two methods, a close similarity in the CA values was obtained. Appropriate amounts $\alpha$-methyldopamine were added to $0.2-1.5$ $\mathrm{ml}$ of deproteinized supernatant as an internal standard, and the volume was adjusted to $8 \mathrm{ml}$ with $0.4 \mathrm{~N}$ perchloric acid containing $0.05 \%$ of EDTA-2Na. The CAs were adsorbed with $200 \mathrm{mg}$ of acid washed aluminum oxide at $\mathrm{pH}$ 8.6, and eluted with $3 \mathrm{ml}$ of $0.2 \mathrm{~N}$ acetic acid-methanol. After lyophilization, the residue was dissolved in $300 \mu$ of the mobile phase of HPLC, and 10-25 $\mu \mathrm{l}$ of the solution was injected into HPLC.

The chromatographic system consisted of a model 6000A pump (Waters Associates, Molford, Mass.), a model U6K universe injector (Waters), and a reverse-phase $\mu$ Bondapak $\mathrm{C}_{18}$ column $(30 \times 0.39 \mathrm{~cm})$. A $4 \mathrm{~cm}$ precolumn, packed with Bondapak $\mathrm{C}_{18} /$ Corasil (Waters), protected the analytical column. The column eluant was monitored with a commercial amperometric detector model LG-4A (Bioanalytical Systems, Inc., West Lafayette, Ind.). The detector potential was set at $0.7 \mathrm{~V}$. The analytical mobile phase, $0.15 \mathrm{M}$ monochloroacetic acid, $2 \mathrm{mM}$ EDTA-2Na, $0.5 \mathrm{mM}$ sodium octyl sulphate and $2 \%$ methanol buffered to $\mathrm{pH} 3.0$, was delivered at a flow rate of $2.0 \mathrm{ml} / \mathrm{min}$. The buffer was filtered $(0.45 \mu \mathrm{m}$, Millipore filter $)$ and degassed under a vacuum 
before the addition of methanol. For assays using GLC-ECD, CAs were assayed according to the method of Kawano et al, ${ }^{11)}$ using $\alpha$-methylnorepinephrine instead of $\alpha$-methyldopamine as an internal standard.

Reagents

Compounds used were L-norepinephrine bitartrate (Sigma), L-epinephrine bitartrate (Sigma), and dopamine (Tokyo chemical Industry Co.). $\alpha$ Methyldopamine and $\alpha$-methylnorepinephrine were generous gifts of the Sterling-Winthrop Research Institute. Other chemicals were of reagent grade.

\section{Statistical methods}

The statistical significance of the differences between mean values of WKY and those of SHR and SHRSP were analyzed using Student's t-test.

\section{RESUlts}

\section{Systolic blood pressure (SBP)}

The SBP of WKY increased gradually, but never exceeded $150 \mathrm{mmHg}$ (Fig. 1). By contrast, the SBP of both SHR and SHRSP increased rapidly after 4 weeks of age (Fig. 1). The SBP of SHRSP and SHR was already significantly higher than that of WKY at both 4 weeks and 7 weeks of age, respectively. Between 7 and 10 weeks of age, the SBP of both SHR and SHRSP exceeded $150 \mathrm{mmHg}$, and these rats were hypertensive. By about 15 weeks of age, the SBP of SHR and SHRSP reached their plateau (181 \pm 4

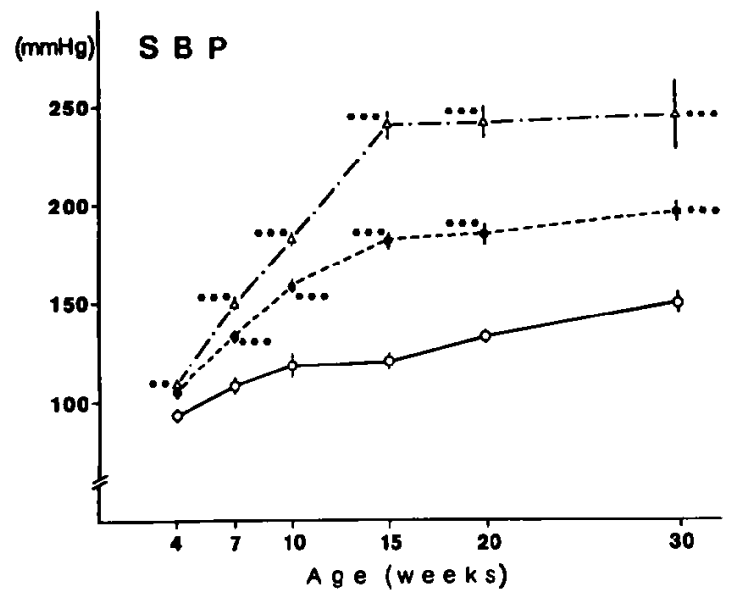

Fig. 1. Changes in systolic blood pressure with age. $\Delta-\cdot-\Delta$ SHRSP, --- SHR, O-O WKY. Each point represents the mean \pm standard error for 5 male rats. ${ }^{*} \mathrm{p}<0.05,{ }^{* *} \mathrm{p}<0.01,{ }^{* * *} \mathrm{p}<0.001$ compared to the value of WKY. 
$\mathrm{mmHg}$ and $240 \pm 7 \mathrm{mmHg}$, respectively) and almost the same high blood pressures were maintained thereafter.

\section{Wet weights of bilateral adrenal glands}

The wet weights of both adrenal glands of all three strains increased rapidly up to 10 weeks of age, then the weights remained at about the same level (Fig. 2). The SHR had the heaviest adrenal glands of these three strains at all ages studied. There were no significant differences in the wet weights of the adrenal glands between the hypertensive and the normotensive strains.

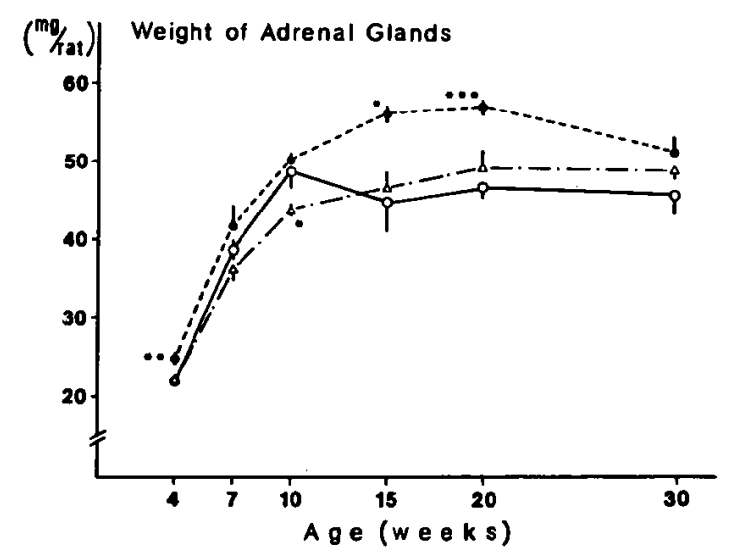

Fig. 2. Changes in wet weight of left and right adrenal glands with age. $\triangle-\cdots \Delta$ SHRSP, --- SHR, O-O WKY. Each point represents the mean \pm standard error for 5 male rats. ${ }^{*} \mathrm{p}<0.05, * * \mathrm{p}<0.01$, ${ }^{* * *} \mathrm{p}<0.001$ compared to the value of WKY.

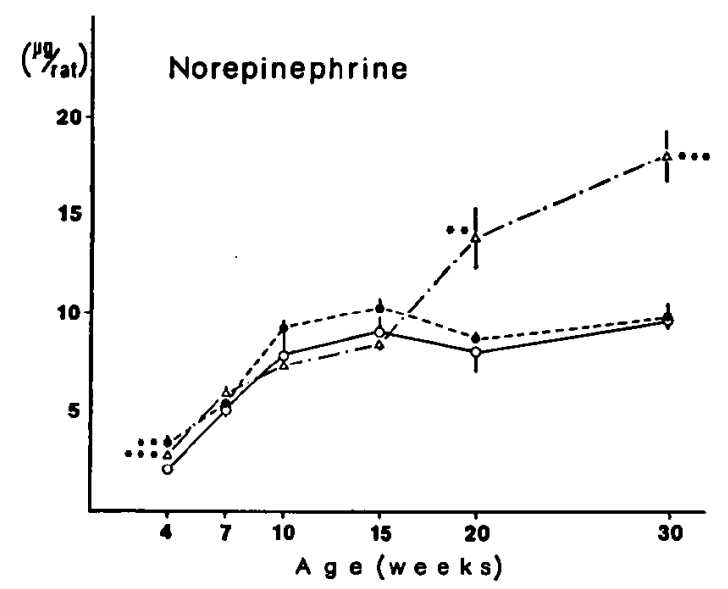

Fig. 3. Changes in norepinephrine contents of the adrenal glands with age. $\triangle-\cdot \triangle$ SHRSP, --- SHR, $\bigcirc-O \mathrm{WKY}$. Each point represents the mean \pm standard error for 5 male rats. ${ }^{*} \mathrm{p}<0.05,{ }^{* *} \mathrm{p}<0.01$, $* * * \mathrm{p}<0.001$ compared to the value of WKY. 


\section{Contents of norepinephrine}

At 4 weeks of age, the NE contents in both SHR and SHRSP were significantly higher than those in WKY (SHR; 3.39 \pm 0.29 , SHRSP; $2.76 \pm 0.05$, WKY; $2.08 \pm 0.07, \mu \mathrm{g} / \mathrm{rat}$ ) (Fig. 3). Thereafter, the NE contents in these three strains followed a course similar to that of the wet weights of the adrenal glands, except for the contents of 20- and 30-week-old SHRSP (Fig. 3). Increases were rapid up to 10 weeks, then much the same levels were maintained until 30 weeks of age. Therefore, the ratio of the contents of NE to $E$ decreased gradually with age.

Unlike the E and the DA contents, the changes in NE contents in SHR were similar to those in WKY, such that the content of NE in the adrenal grands was essentially the same when age-matched WKY and SHR were compared.

Only in SHRSP did the NE contents increase rapidly after the development of hypertension, while no change in the NE contents was seen in SHR and WKY. The NE content in SHRSP was about twice that of SHR and WKY, when compared at 30 weeks of age (Fig. 3).

\section{Contents of dopamine}

The DA content in SHRSP was significantly lower than in WKY at 4 weeks of age (SHRSP; 0.086 \pm 0.005 , WKY; 0.151 $\pm 0.015, \mu \mathrm{g} / \mathrm{rat}$ ) (Fig. 4). The contents in SHRSP, however, increased continuously throughout the period studied. The DA content in SHRSP was significantly higher than in WKY at 20 weeks of age, and finally exceeded even the level of SHR at 30 weeks of age.

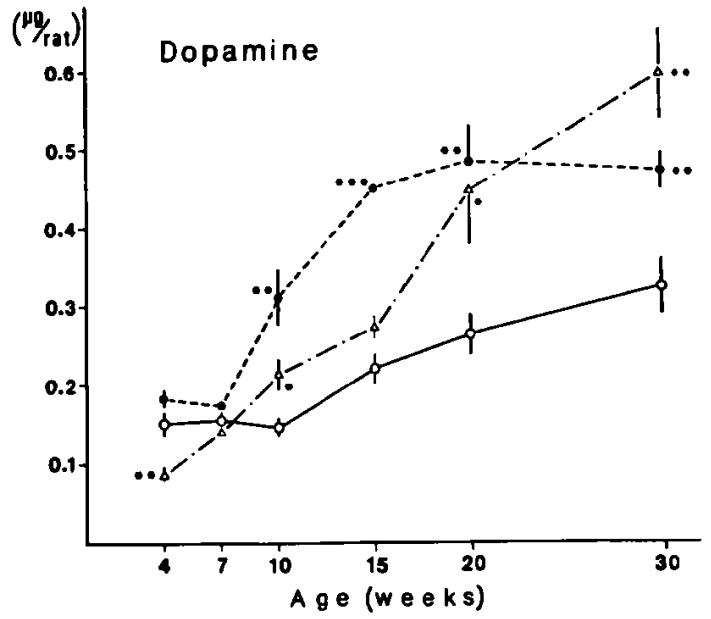

Fig. 4. Changes in dopamine contents of the adrenal glands with age. $\triangle-\cdot-\triangle$ SHRSP, -- SHR, O-O WKY. Each point represents the mean \pm standard error for 5 male rats. ${ }^{*} p<0.05,{ }^{* *} p<0.01,{ }^{* * *} \mathrm{p}<$ 0.001 compared to the value of WKY. 


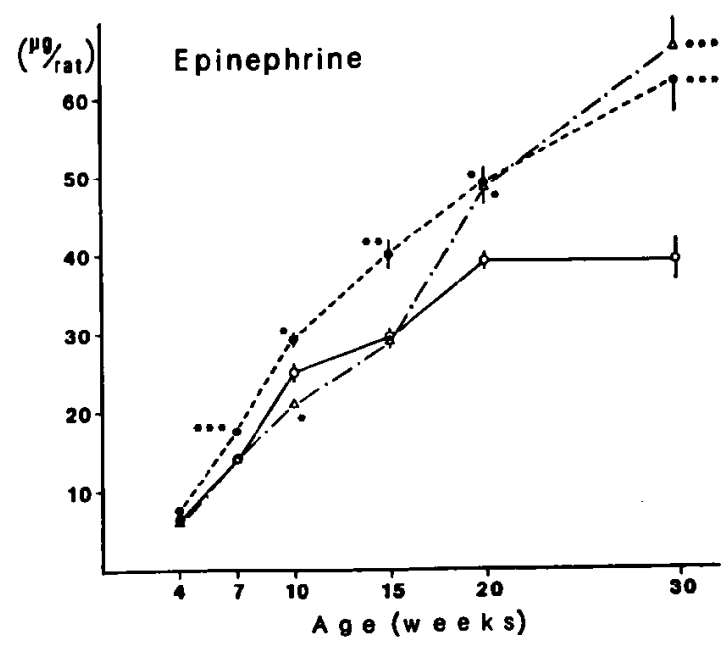

Fig. 5. Changes in epinephrine contents of the adrenal glands with age. $\triangle-\cdot-\Delta$ SHRSP, ---O SHR, O-O WKY. Each point represents the mean \pm standard error for 5 male rats. ${ }^{*} p<0.05,{ }^{* *} p<0.01,{ }^{* * *} p<$ 0.001 compared to the value of WKY.

The DA content in SHR was similar to WKY at 4 and 7 weeks of age, but the level began to increase at around 7 weeks of age. It increased rapidly until 15 weeks of age, then was maintained at about twice the level seen in the WKY (Fig. 4).

\section{Contents of epinephrine}

The $\mathrm{E}$ content, which is the largest of the adrenal CA contents, increased proportionally to the age during most of the intervals studied in each strain (Fig. 5). The E content in SHR was significantly greater than that in WKY at each age studied, except at 4 weeks. Although the E contents in SHRSP were similar to those in WKY up to 15 weeks of age, these levels began to increase more rapidly than those in WKY. Consequently, after 20 weeks of age, the $\mathrm{E}$ contents in SHRSP were significantly higher than those in WKY and reached values similar to those seen in the SHR (Fig. 5).

\section{Discussion}

By about 15 weeks of age, the SBP of both the SHR and the SHRSP reaches a plateau and remains stable thereafter. On the other hand, the incidence of stroke increased in the SHRSP in our colony from around 15 weeks of age, when their SBP had already reached $240 \mathrm{mmHg}$. At 20 weeks of age, almost all rats had cerebrovascular lesions. Therefore, it is necessary to compare the hypertensive strains (SHR and SHRSP) and the normotensive 
strain (WKY) up to 15 weeks of age in order to discuss the pathogenesis of the development of hypertension. Also it is necessary to compare the strokeprone strain (SHRSP) and the stroke-resistant strains (SHR and WKY) after 15 weeks of age to discuss the etiology of cerebral stroke.

The major differences up to 15 weeks of age between the hypertensive strains and normotensive strain were as follows: 1) At 4 weeks of age, the NE contents in the hypertensive strains were significantly higher than in the normotensive strain. 2) During the development of hypertension, the DA contents in the hypertensive strains increased rapidly, whereas those in the normotensive strain did not change to any great extent.

It has been proposed that the facilitation of peripheral sympathetic NE neurons, related to central abnormalities at a young age, may act as a trigger in the development of hypertension in SHR, causing the constriction of peripheral arteries and an adaptive structural change of vessel wall due to the hypertension. ${ }^{12)}$ This facilitation has been verified in young SHR by using the parameters of the $\mathrm{NE}$ concentration in the serum, ${ }^{13)}$ dopamine- $\beta$-hydroxylase activity in the serum ${ }^{14}$ and the mesentric artery, ${ }^{15)}$ and tyrosine hydroxylase activity in the celiac ganglion. ${ }^{16}$ ) The high contents of adrenal NE in SHRSP and SHR at 4 weeks of age, observed in the present study, provide additional evidence for this facilitation. This observation was not consistent with the deficient activity of CA synthesizing enzymes in the adrenal glands of 4-week-old SHR reported by Grobecker et al. ${ }^{13)}$ It should be noted that only the NE level was consistent with this facilitation of the peripheral sympathetic NE neurons rather than $E$, which is concentrated in the adrenal glands.

Lutold et $\mathrm{al}^{71}$ reported that the celiac ganglion of the 4-week-old SHR contained more NE and that there was a deficiency in the SIF cell-dopaminergic system as compared with ganglion from WKY. The DA released from sympathetic ganglion SIF cells suppresses ganglionic transmission. ${ }^{17}$ Thus, the deficient release of DA from the SIF cells would elevate postganglionic sympathetic activity and vasoconstriction would ensue. In the present study, the NE content was elevated and the DA content suppressed in the adrenal glands in SHRSP at 4 weeks of age, as observed by Lutold et al ${ }^{71}$ in the celiac ganglion of SHR at the same age. Although the physiological function of the adrenal DA is unknown, the adrenal gland is, in effect, a sympathetic ganglion in which the postganglionic neurons have lost their axons. Therefore, the suppressed DA content in the adrenal glands may contribute to the facilitation of the adrenal NE cells.

A distinctive difference after 15 weeks of age between the stroke-prone strain and the stroke-resistant strains was found in the abnormal increase in 
levels of the three CAs in the SHRSP. These abnormal increases of CAs in SHRSP may relate to the development of cerebral stroke, or may be a secondary effect resulting from stress or other factors related to stroke.

In the early investigations of SHR, it was demonstrated that the adrenal NE content in the adult SHR was almost twice that seen in the WKY.3),4) In our observations, however, there was no significant difference between SHR and WKY in the adrenal NE contents, except at 4 weeks of age. These discrepancies are attributed to mating with stroke-resistant rats whose SBP was under $200 \mathrm{mmHg}$ and which were selected from among SHR for long term study, because the NE content in SHRSP was almost twice that in WKY at 20 and 30 weeks of age.

Conversely, the content of $\mathrm{E}$ in SHR was much higher than that in WKY, at every age except 4 weeks. Majewski and Rand $^{18)}$ proposed that the epinephrine-mediated hypertensive mechanisms were the result of action of the facilitatory prejunctional $\beta$-adrenoceptors. They speculated that the hypertension in SHR may be derived from the circulating $E$ secreted from the adrenal medulla. Nevertheless, these elevated $\mathrm{E}$ contents in the adrenal glands of SHR may not be related to the development of hypertension, but rather to a "natural" hypersensitivity to stress, ${ }^{19,20)}$ because the $\mathbf{E}$ contents in SHRSP were similar to those in WKY up to 15 weeks.

We have no direct explanation as to why the DA contents in SHR increased rapidly after 7 weeks of age and reached about twice the level of those in WKY. However, the increase in the DA contents may, a compensate for an increase in the $\mathrm{E}$ contents.

In conclusion, the facilitation of peripheral sympathetic NE neurons was also verified in observations of adrenal NE contents in both SHRSP and SHR at 4 weeks of age. SHRSP was clearly distingushed from SHR by comparing the adrenal CA contents of each strain. The contents of all three CAs in SHRSP were similar to those of WKY during the development of hypertension, while the contents of $\mathbf{E}$ and DA in the SHR were much higher than those in the WKY. Only in SHRSP did the contents of all three CAs increase rapidly after the development of hypertension. These rapid increases may be related to apoplexy or stroke.

\section{Agknowledgment}

We thank Mariko Ohara for critical reading of the manuscript. 


\section{REFERENGES}

1. Nagatsu T, Mizutani K, Nagatsu I, Umezawa H, Matsuzaki M, Takeuchi T: Catecholamine synthetic enzymes of spontaneously hypertensive rats and microbial hypotensive products. Mol Cell Biochem 1 : 107, 1973

2. Morisawa $\mathrm{T}$ : On the noradrenaline reaction of the adrenal medulla in experimental hypertensive rats, especially in spontaneously hypertensive rats. Jpn Circulat J 32: 161, 1968

3. Ozaki M, Suzuki Y, Yamori Y, Okamoto K: Adrenal catecholamine content in the spontaneously hypertensive rats. Jpn Circulat J 32: 1367, 1968

4. Tabei R, Morisawa T, Maruyama T, Suzuki Y: Noradrenaline in the adrenal medulla of spontaneously hypertensive rats. Jpn J Constit Med 30: 153, 1967 (in Japanese)

5. Nagatsu T, Kato T, Hashimoto Y, Numata (Sudo) Y, Yamori Y, Okamoto K: Dopamine$\beta$-hydroxylase activity in stroke-prone spontaneously hypertensive rats. Experientia 34: 305, 1978

6. Ozaki M: Metabolism of monoamines in spontaneously hypertensive rats (2). Jpn J Constit Med 30: 155, 1967 (in Japancse)

7. Lutold BE, Karoum F, Neff NH: Deficient dopamine metabolism in the celiac ganglion of spontaneously hypertensive rats. Circulat Res 44: 467, 1979

8. Imai $K$, Sugiura $M$, Tamura $Z$ : Catecholamine in rat tissues and serum determined by gas chromatographic method. Chem Pharm Bull 19: 409, 1971

9. Da Prada $M, Z$ Zürcher $G$ : Simultaneous radioenzymatic determination of plasma and tissue adrenaline, noradrenaline and dopamine within the femtomole range. Life Sci 19: 1161, 1976

10. Kissinger PT, Refshauge CJ, Dreiling R, Adams RN: An electrochemical detector for liquid chromatography with picogram sensitivity. Anal Lett 6: 465, 1973

11. Kawano T, Niwa M, Fujita Y, Ozaki M, Mori K: An improved method for analysis of catecholamines-gas-liquid chromatography (GLC) equipped with electron-capture detector. Jpn J Pharmacol 28: 168, 1978

12. Haeusler G, Finch L, Thoenen H : Central adrenergic neurons and the initiation and development of expreimental hypertension. Experientia 28: 1200, 1972

13. Grobecker H, Roizen MF, Weise V, Saavedra JM, Kopin IJ : Sympathoadrenal medullary activity in young, spontaneously hypertensive rats. Nature 258: 267, 1975

14. Nagatsu T, Kato T, Numata (Sudo) Y, Ikuta K, Umezawa H, Matsuzaki M, Takeuchi T: Serum dopamine $\beta$-hydroxylase activity developing hypertensive rats. Nature 251 : 630, 1974

15. Nagatsu T, Ikuta K, Numata (Sudo) Y, Kato T, Sano M, Nagatsu I, Umezawa H, Matsuzaki $M$, Takeuchi $T$ : Vascular and brain dopamine 3 -hydroxylase activity in young spontaneously hypertensive rats. Science 191: 290, 1976

16. Nakamura K, Nakamura $\mathrm{K}$ : Selective activation of sympathetic ganglia in young spontaneously hypertensive rats. Nature 266: 265, 1977

17. Willems JL: Dopamine-induced inhibition of synaptic transmission in lumbar paravertebral ganglia of the dog. Naunyn Schmiedebergs Arch Pharmacol 279: 115, 1973

18. Majewski $H$, Rand MJ: Adrenaline-mediated hypertension: a clue to the antihypertensive effect of $\beta$-adrenoceptor blocking drugs? Trends Pharmacol Sci 17: 24, 1981

19. McCarty R, Kopin IJ: Alterations in plasma catecholamines and behavior during acute stress in spontaneously hypertensive and Wistar-Kyoto normotensive rats. Life Sci 22: 997, 1978

20. Chiuch CC, Kopin IJ: Hyperresponsivity of spontaneously hypertensive rat to indirect measurement of blood pressure. Am J Physiol 234: H690, 1978 